\title{
Effect of Surfactant Concentration on the Long-Term Properties of a Colloidal Chemical, Biological and Radiological (CBR) Decontamination Gel
}

\author{
Alban Gossard *, Fabien Frances, Camille Aloin, Clara Penavayre, Nicolas Fabrègue and Célia Lepeytre \\ CEA, DES, ISEC, DMRC, Univ Montpellier, Marcoule, France; fabien.frances@cea.fr (F.F.); \\ camille-marie.aloin@cea.fr (C.A.); clara.penevayre@cea.fr (C.P.); nicolasj.fabregue@gmail.com (N.F.); \\ celia.lepeytre@cea.fr (C.L.) \\ * Correspondence: alban.gossard@cea.fr; Tel.: +33-4-66-33-91-34
}

check for

updates

Citation: Gossard, A.; Frances, F.; Aloin, C.; Penavayre, C.; Fabrègue, N.; Lepeytre, C. Effect of Surfactant Concentration on the Long-Term Properties of a Colloidal Chemical, Biological and Radiological (CBR) Decontamination Gel. Fluids 2021, 6, 410. https://doi.org/10.3390/ fluids 6110410

Academic Editor: Rajinder Pal

Received: 25 October 2021

Accepted: 9 November 2021

Published: 12 November 2021

Publisher's Note: MDPI stays neutral with regard to jurisdictional claims in published maps and institutional affiliations.

Copyright: (c) 2021 by the authors. Licensee MDPI, Basel, Switzerland. This article is an open access article distributed under the terms and conditions of the Creative Commons Attribution (CC BY) license (https:// creativecommons.org/licenses/by/ $4.0 /)$.

\begin{abstract}
Chemically, biologically, or radiologically contaminated surfaces can be treated using colloidal "vacuumable" gels containing alumina particles as a thickening agent, decontaminating solutions to inhibit/eliminate biological and chemical contaminants, and Pluronic PE 6200 as a surfactant to adjust the gel's physicochemical properties. These gels have been shown to remain efficient even after prolonged storage. In the present study, the properties of gels with different surfactant concentrations were monitored over several months using rheological analyses, contact angle measurements, and ion chromatography. Results show that the surfactant reacts with the hypochlorite ions in the decontaminating solution. This leads to sedimentation, which modifies the rheological properties of the gel. Increasing the surfactant concentration ensures the physicochemical properties of the gel are preserved for longer, but because the surfactant reacts with the hypochlorite ions, the concentration of the latter decreases drastically and thus so do the decontamination properties of the gel. There is therefore a trade-off between the efficiency of the gel against chemical and biological contamination at given time and how long its physicochemical properties are preserved, with the optimal balance depending on its intended use.
\end{abstract}

Keywords: CBRN; gel; decontamination; surfactant; rheology

\section{Introduction}

Colloidal gels are complex fluids that can be used to decontaminate solid surfaces [1-8]. These gels consist of mineral colloidal particles (typically silica or alumina) dispersed and suspended in a decontaminating solution. Surfactants are also added to gel formulations to adjust their physicochemical properties. These gels can be sprayed on contaminated surfaces (ceiling, walls, floors), and their specific rheological properties ensure they adhere, even on vertical surfaces, allowing prolonged contact between the surface and the decontaminating solution contained in the gel. Once spread, the gels rapidly trap labile contamination. If necessary, the composition of the gel can be adjusted to attack the first few microns of the surface to release incrusted or fixed contaminants, most notably on metallic surfaces. The gel with the contamination trapped inside then dries and finally crumbles, just as classical colloidal suspensions do, into a non-powdery solid that is readily removed by brushing or vacuuming. These "vacuumable gels" can thereby be used to decontaminate solid surfaces without generating any secondary liquid effluent, i.e., without any post-treatment required.

Initially developed for the radiological decontamination of metallic surfaces $[1,7,8]$, this technology has been expanded to applications such as depollution [3] and the elimination of sticky bitumen layers [9]. Similar gels have also been formulated for the chemical, biological, and radiological (CBR) decontamination of solid surfaces [2,4], with hypochlorite ions and sodium hydroxide added to the decontaminating solution to treat biological 
and chemical contamination. Solid contaminants such as radioactive or biological particles are absorbed, while chemical and ionic nuclear contaminants are solubilized and neutralized either by oxidation (hypochlorite) or by hydrolysis (sodium hydroxide).

While this process is very effective, the gels could suffer of conservation issues. Their stability is governed by DLVO (Derjaguin-Landau-Verwey-Overbeck) theory, which considers the interactions between particles in solution as the sum of Van der Waals attractive forces and electrostatic repulsion [10-12]. The gels typically destabilize over time and even sediment with the formation of a liquid supernatant phase on the surface. This affects rheological properties of the gel. In CBR gels, moreover, the concentration of hypochlorite ions has been observed to decrease over time [4]. This was found to have no impact on the neutralization of Bacillus thuringiensis and of Bacillus anthracis spores but may be problematic under other circumstances.

To better understand the microstructural evolution of these gels during aging and to overcome these problems, we have studied the long-term evolution of the rheological and chemical properties of a series of colloidal CBR gels. We first focus on the aging of a CBR gel previously described by Lepeytre et al. [4], and hypotheses based on the degradation of the surfactant in the decontaminating solution are advanced to explain the evolution of its rheological properties. To confirm these hypotheses, the surfactant concentration was increased, and the effects of this modification are highlighted by rheological analyses, contact angle measurements, and ion chromatography to monitor the nature of the chlorinated species in solution. A mechanism is finally proposed to explain the role of the surfactant concentration on the rheological and chemical aging of CBR gels.

\section{Materials and Methods}

\subsection{Materials}

Alumina was purchased as Aeroxide Alu C (specific surface area, $100 \mathrm{~m}^{2} \cdot \mathrm{g}^{-1}$ ) from Evonik Industries (Essen, Germany). Pluronic PE 6200 was purchased from BASF (Ludwigshafen, Germany) and iron oxide (Ferroxide 212M) from Rockwood (Beltsville, MD, USA). Sodium hydroxide was purchased from Sigma Aldrich (St. Louis, MO, USA) and bleach (sodium hypochlorite) from Fisher (Hampton, NH, USA).

\subsection{Gel Preparation and Aging}

The process used to prepare the CBR gel investigated here has been described in detail by Lepeytre et al. [4]. Briefly, the gels were obtained by mixing $14 \mathrm{wt} . \%$ of Aeroxide Alu C alumina, as a thickening agent, $x$ wt.\% $(x=0-5)$ of Pluronic PE 6200, as a surfactant, and $0.1 \mathrm{wt} . \%$ of iron oxide as a coloring pigment, with $(85.9 \mathrm{x}) \mathrm{wt} . \%$ of decontaminating solution ( $0.5 \mathrm{M} \mathrm{NaOH}$ with hypochlorite ions). First, the Pluronic PE 6200 was diluted in the decontaminating solution using a three-blade stirrer at $200 \mathrm{rpm}$ for $5 \mathrm{~min}$. The iron oxide and alumina particles were then gradually added, and the stirring speed was increased up to $500 \mathrm{rpm}$ as the viscosity increased. Stirring was finally maintained for $5 \mathrm{~min}$ to homogenize the gel. The gels obtained are referred to as SFx, where $x$ is the surfactant weight percentage, with as-prepared gels referred to as "fresh". To evaluate the effects of storage, samples of SF1 CBR gel were stored for between 18 and 36 months in the dark at room temperature.

\subsection{Characterizations}

The $\mathrm{pH}$ of the gels was measured using a glass combination electrode connected to a WTW pH meter calibrated at $\mathrm{pH} 4.01,7.00$, and 9.18. Rheological measurements were performed with a Discovery HR-1 rheometer (TA Instruments) with a parallel plate geometry ( $40 \mathrm{~mm}$ diameter, $1 \mathrm{~mm}$ gap). The gels were manually homogenized before the rheological measurements. This manual homogenization does not affect the rheological analyses because the gels recover their properties almost immediately after shearing. The stability of the suspensions was checked to verify that no sedimentation would occur during the rheological experiments. In the first experiment, the stress of the gels was 
measured using a flow ramp test with shear rates decreased from $100 \mathrm{~s}^{-1}$ to $0.01 \mathrm{~s}^{-1}$ over $60 \mathrm{~s}$. In the second experiment, shear stress was measured as a function of strain at a low constant shear rate $\left(0.0067 \mathrm{~s}^{-1}\right)$. To evaluate the sedimentation of the gels, several samples were prepared and stored in a closed vessel in the dark at room temperature. At regular intervals and without agitation, any supernatant that had formed was extracted using a pipette and weighed before being delicately replaced in the vessel for further measurements. The wettability of decontaminating solutions (i.e., without alumina) with different concentrations of surfactant was assessed by measuring the contact angle of a drop of the solution on a ceramic tile (identical to the ones used in the Paris metro) using a Tracker automatic drop tensiometer (Teclis Scientific, Civrieux d'Azergues, France). The contact angle was determined by depositing drops of solution with a constant volume on a ceramic tile. After $1 \mathrm{~min}$, to reach equilibrium, a picture was taken and the contact angle was directly measured on this picture. After three measurements, performed successively, an average was taken to obtain the contact angle value. The evolution of the chloride concentration in these decontaminating solutions was measured by ion chromatography using a Metrohm 881 Compact IC pro device (Herisau, Switzerland), while the concentration of hypochlorite ions was determined using an in-house titration method (described in Appendix A).

\section{Results and Discussion}

\subsection{Physicochemical Characterization and Stability}

The SF1 CBR gel has already been shown by Lepeytre et al. to have satisfactory decontamination factors for chemical, biological, and radiological contaminants even after two years of aging [4]. In the present study, the evolution of the gel's physicochemical properties was monitored up to 36 months. To ensure that the gel can be applied by spraying, the gel should behave as a shear-thinning fluid as it passes through the spraying nozzle, and it should have a sufficiently high yield stress to remain in place once spread in a millimeter-thick layer on a vertical surface. Sedimentation of the alumina particles is also undesirable as this would disrupt the viscoelastic network.

\subsubsection{Macroscopic Aging of CBR Gel SF1}

A pot of SF1 gel was stored for 9 months and regularly inspected. No changes were observed for the first 3 months, but thereafter, the gel was seen to destabilize and a supernatant liquid appeared. The evolution of the weight percentage of the supernatant (relative to the total mass of the gel) as a function of time is shown in Figure 1.

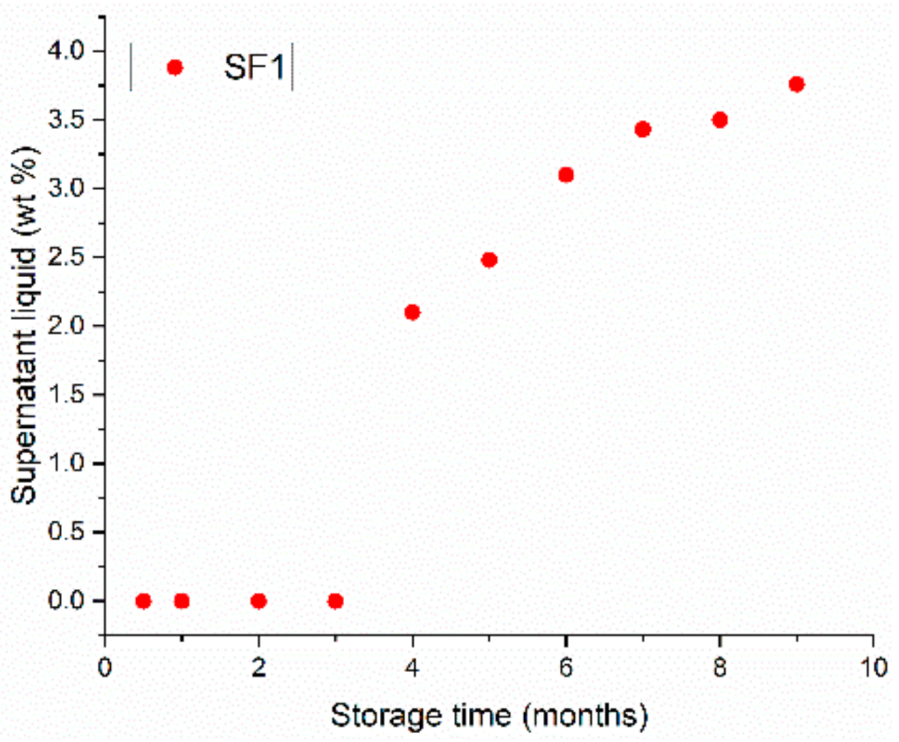

Figure 1. Weight percentage of supernatant as a function of storage time of a CBR gel with 1 wt.\% surfactant (gel SF1). 
The proportion of supernatant increased from about $2 \mathrm{wt} . \%$ at 4 months of storage up to $3.7 \mathrm{wt}$ \% after 9 months, reflecting changes in the internal physicochemical properties of the colloidal suspension.

\subsubsection{Rheological Properties of the Gel during Aging}

Rheological analyses were performed to better understand how the gels evolve over time. Figure 2a shows the flow curves of the SF1 gel measured at different aging times up 36 months alongside the corresponding data for a fresh sample of SF0 CBR gel. Note that to avoid a concentration effect due to the formation of supernatant, the samples were all manually homogenized before analysis.
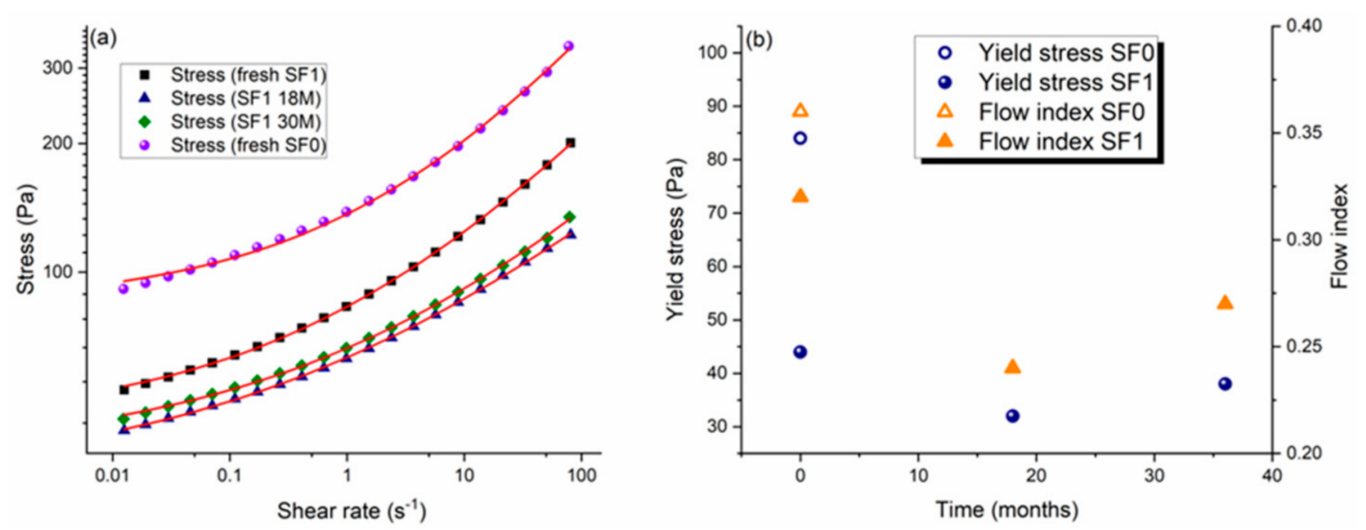

Figure 2. Influence of aging time on the rheological properties of a CBR gel with $1 \mathrm{wt}$ \% surfactant (gel SF1). (a) Shear stress as a function of shear rate with experimental data points and lines of best fit using the Herschel-Bulkley model for aged samples of SF1 gel and a fresh sample of SF0 gel. (b) Yield stress and flow index as a function of storage time obtained from the fits using the Herschel-Bulkley model.

The rheological behavior of the gels can be modeled using the Herschel-Bulkley model [13-15]:

$$
\tau=\tau_{y}+K \dot{\gamma}^{n}
$$

where $\tau$ is the shear stress applied to the gel, $\tau_{y}$ is the yield stress of the gel, $K$ is its consistency (a structure-dependent proportionality constant), and $n$ is the flow index, which quantifies the level of deviation from Newtonian behavior. The values of the yield stress and flow index obtained by fitting the data in Figure 2a are plotted in Figure $2 \mathrm{~b}$.

The SF1 and SF0 CBR gels both have a yield stress and show shear thinning with $n<1$ for at least 36 months. This behavior is typical of colloidal mineral suspensions in which the particles self-organize into streamlined assemblies that readily flow under shearing, decreasing the bulk viscosity of the suspension [16,17]. Shear-thinning is a key property for decontaminating gels as they need to be liquid enough to be pumped out initially from their storage tank and then pass easily through the spraying nozzle without clogging. An appropriate yield stress is also necessary. The yield stress is a critical value specific to the internal interactions between the particles and the surfactant. When the shear stress in the material is lower than the yield stress, the gel behaves as an elastic solid, while above this threshold the gel flows like a liquid. In other words, the gel only flows if a sufficiently high stress is applied. For these CBR gels, the yield stress has to be more than $10 \mathrm{~Pa}$ to ensure a millimeter layer does not flow down under gravity but cannot be too high because the gel has to be sprayable with standard industrial devices [1,2].

Without surfactant and in basic media (as in the SF0 CBR gel), the alumina particles aggregate strongly and form an "attractive" gel [18]. The addition of surfactant strongly affects the flow behavior of the gel, as Pluronic PE 6200, a wetting agent, disperses the suspended solid particles and limits aggregation. As a result, the particles flow more easily, reducing the viscosity of the gel and increasing the level of shear-thinning. Furthermore, 
although adding $1 \mathrm{wt} . \%$ surfactant reduces the yield stress of the gel, the resulting value (44 $\mathrm{Pa}$ ) is still compatible with the application process.

Figure 2a shows that the rheological properties of the SF1 CBR gel vary over time. The yield stress and flow index decrease initially for a few months and then increase in a second stage. These variations probably reflect internal physicochemical modifications, as illustrated by the stress-strain curves measured after different aging times (Figure 3).

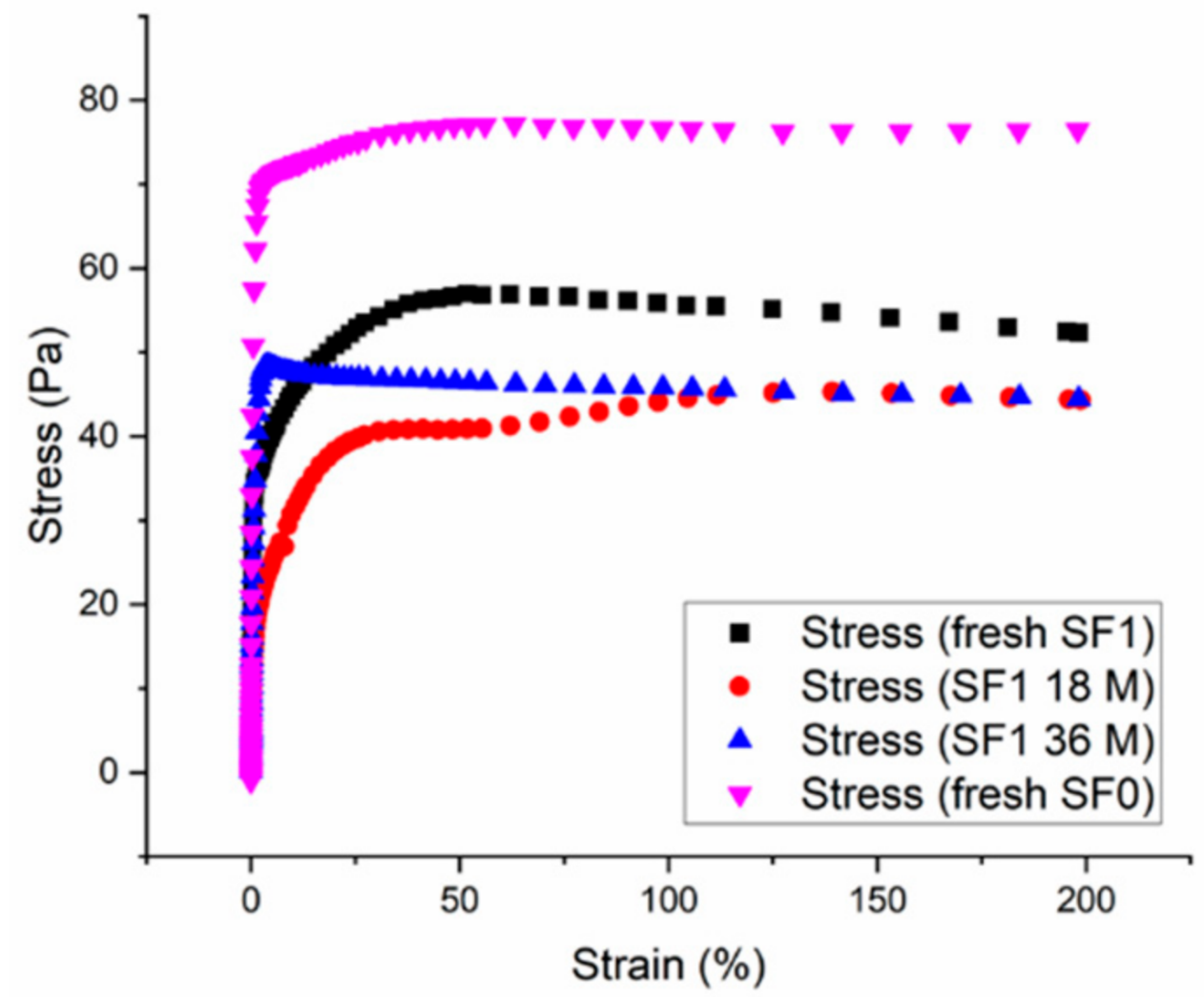

Figure 3. Shear stress versus strain at a constant shear rate of $0.0067 \mathrm{~s}^{-1}$ for samples of fresh SF0 CBR gel (no surfactant) and of SF1 CBR gel (1 wt.\% surfactant) either fresh or aged for 18 or 36 months.

At low strain rates, the stress increases linearly as the gel behaves as a solid and deforms elastically until the yield stress is reached and the gel begins to flow like a liquid [16]. Because these data were measured at a very low shear rate, the values of the yield stress obtained can be compared with those obtained by fitting the data in Figure $2 \mathrm{a}$ to the Herschel-Bulkley model. The latter are a bit lower because the yield stress is defined at a shear rate of zero, but the same trends are observed with both approaches, namely that adding surfactant reduces the yield stress of the gel and that the yield stress has a U-shaped time evolution.

The curve profiles in Figure 3 provide complementary information. In the gel without surfactant (SF0), the stress increases sharply at the lowest strain rates and the elastic domain is very limited, indicating that the Van der Waals interactions between the initially aggregated alumina particles are weak and easily broken. In the gel with surfactant (SF1), the alumina particles are initially less aggregated but the elastic domain extends to higher strain rates (Figure 3, CBR-Gel SF1). This is because the surfactant, Pluronic PE 6200, is adsorbed by hydrogen bonding at the surface of the alumina particles through its polyethylene glycol groups [19-21], leaving the hydrophobic polypropylene glycol group "free" in solution as represented on Figure 4. Pluronic PE 6200 thus disperses alumina particles in solution but also creates a form of secondary network through intermolecular hydrophobic interactions, making the gel more elastic. 


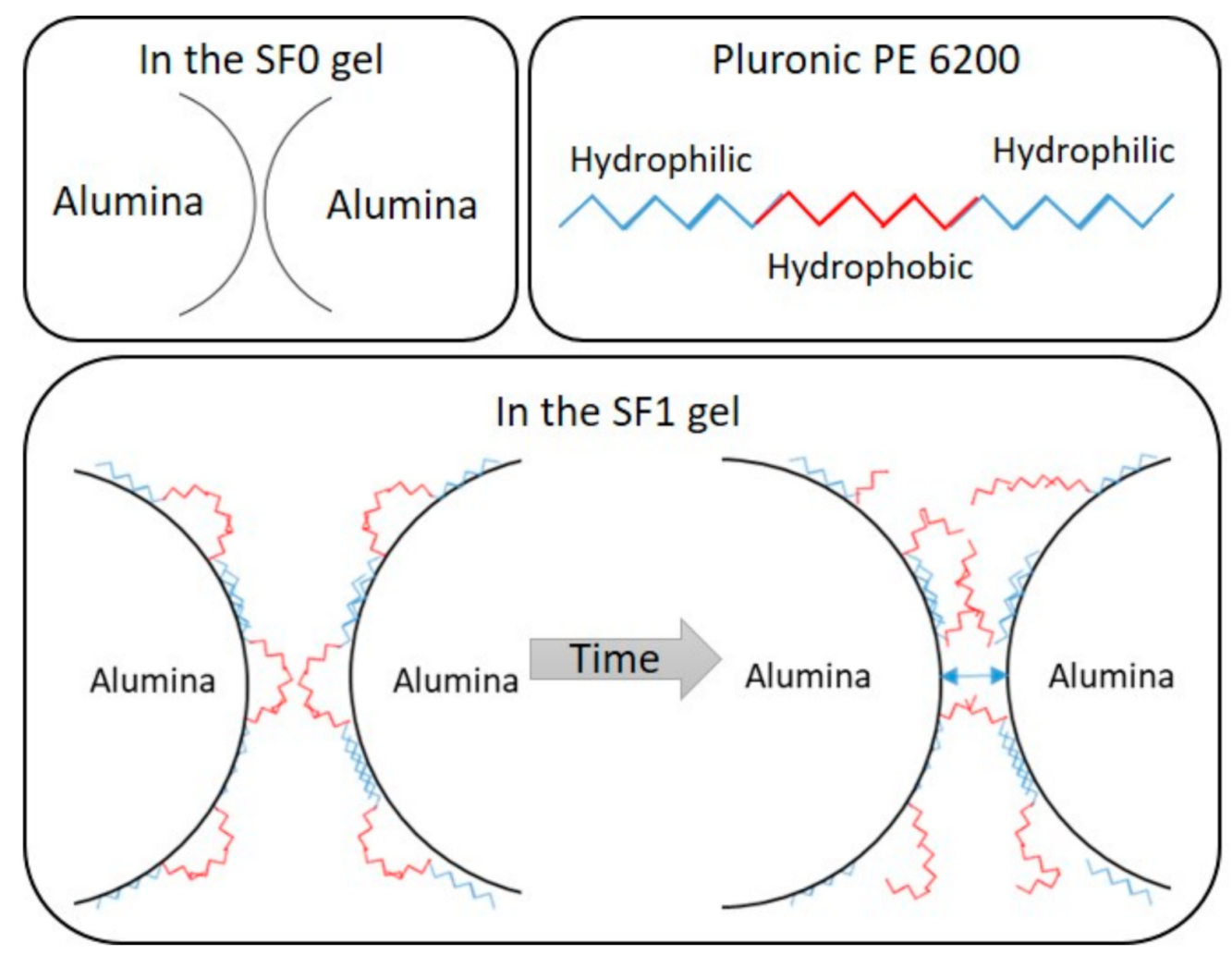

Figure 4. Schematic representation of the alumina-surfactant interaction in the gels.

The aged SF1 gel samples have more limited elastic domains (Figure 3), probably because of the degradation of the surfactant. As described by the manufacturer (BASF), Pluronic PE surfactants tend to degrade in alkaline media and the presence of hypochlorite ions in the decontaminating solution may accelerate this process, notably by oxidation of the ether function. We assume that the degradation process involves two steps. The surfactant molecules first start to degrade but remain partly adsorbed on the surface of the alumina particles such that they remain dispersed, but the secondary hydrophobic network is weakened and the gel becomes less elastic. The viscosity and the yield stress of the gel decrease and the gel flows more easily. As the surfactant degrades further, the interaction between alumina particles come to predominate, and the elasticity of the gel becomes negligible (see Figure 4). These alumina particles' interactions induce a stiffening of the gel, as observed for the SF0 gel, and thus an increase of the yield stress. The fact that the yield stress and flow index increase only slightly suggests that the presence of residual surfactant fragments on the surface of the particles may still limit the aggregation of alumina particles and influence the behavior of the suspension.

To conclude, the evolution of the SF1 gel's rheological properties is consistent with the expected degradation of the Pluronic PE 6200 surfactant in basic media and/or the presence of hypochlorite ions that may oxidize its ether bond. Additional formulations with higher surfactant contents were therefore studied as a potential solution to this problem.

\subsection{Impact of the Surfactant Concentration on the Properties of the Gel}

3.2.1. Impact of the Surfactant Concentration on Gel Sedimentation

Figure 5 highlights the influence of the surfactant concentration of the sedimentation of CBR gels. 


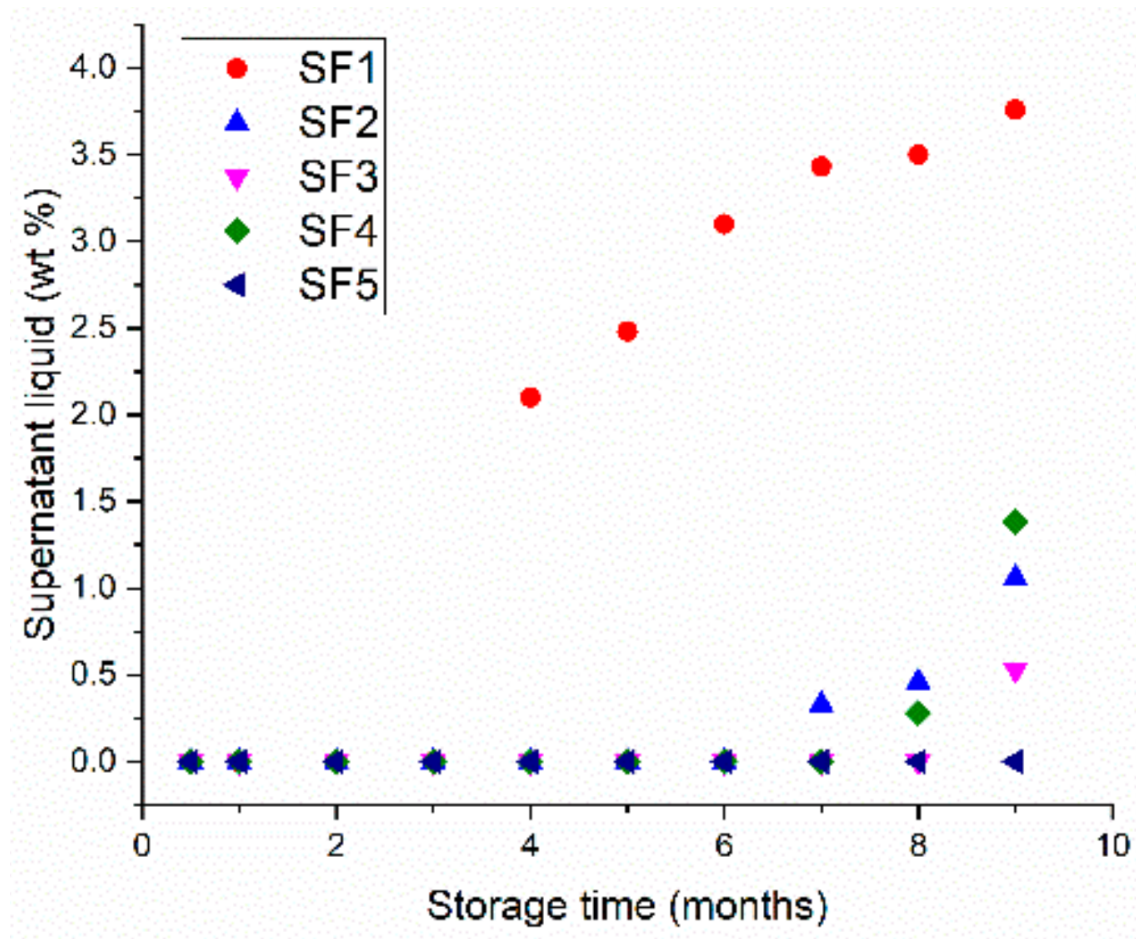

Figure 5. Weight percentage of supernatant as a function of storage time for CBR gels with 1-5 wt.\% surfactant (SF1-SF5).

The higher the surfactant content of the gel is, the longer the sedimentation is delayed for and the smaller the volume of supernatant that forms. The gels with 2, 3, and 4 wt.\% remain stable with no supernatant for 6 months, while the SF5 gel remains stable for more than 9 months. One possible explanation is that the higher surfactant concentration ensures the secondary hydrophobic network is maintained for longer, even after some of the surfactant has degraded in the alkaline environment of the gel. The increase in the surfactant concentration from $1 \mathrm{wt} . \%$ to $2 \mathrm{wt} . \%$ strongly improves the stability of the gel because the secondary network is significantly reinforced. Then, this influence is less marked for more than $2 \mathrm{wt} . \%$ because the gel microstructure is not very affected anymore.

\subsubsection{Impact of the Surfactant Concentration on the Rheological Properties of the Gel}

Figure 6 shows how increasing the surfactant concentration modifies the rheological properties of the CBR gel.
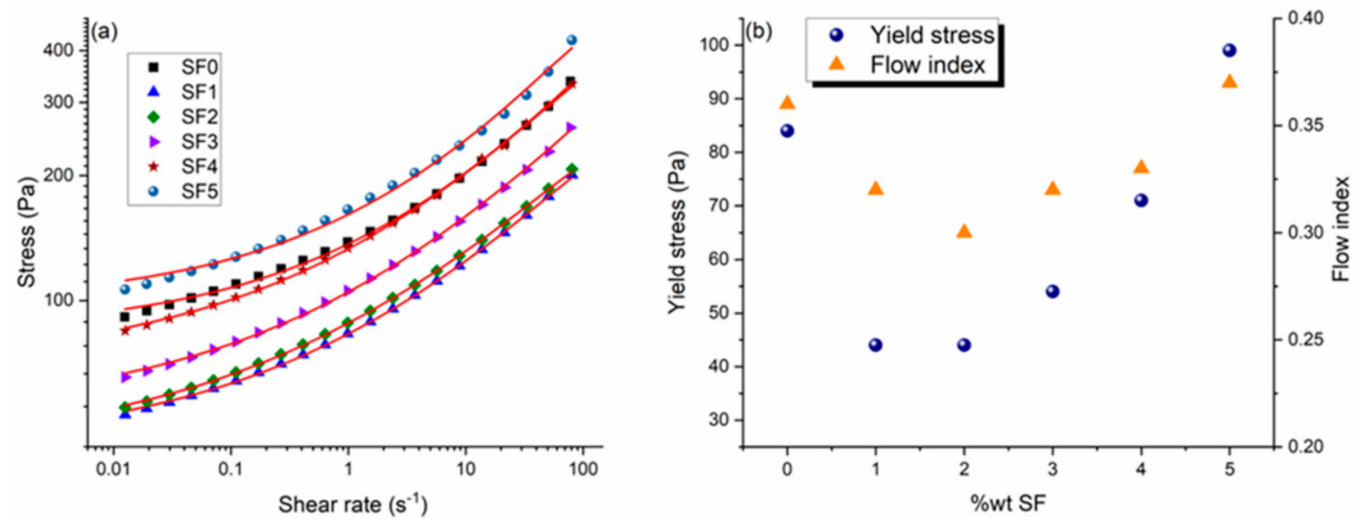

Figure 6. Influence of the surfactant concentration (0-5 wt.\%) on the rheological properties of the CBR gel: (a) shear stress as a function shear rate with experimental data points and lines of best fit using the Herschel-Bulkley model. (b) Yield stress and flow index as a function of the surfactant concentration obtained from the fits using the Herschel-Bulkley model. 
As mentioned above, adding $1 \mathrm{wt} . \%$ of surfactant reduces both the viscosity and yield stress of the gel because Pluronic PE 6200 disaggregates the alumina particles. However, adding more than $2 \mathrm{wt} . \%$ surfactant leads to a stiffening of the gel, as reflected by increases in the yield stress and flow index. These results are consistent with the proposed formation of a hydrophobic network of alumina particles connected by adsorbed surfactant molecules, whose strength increases with the surfactant concentration. The viscoelastic properties of the gel are thus a combination of those of the network of alumina particles and of the organization of the surfactant's organic chains.

Figure 7 compares the stress-strain curves of CBR gel samples with $0-5$ wt.\% surfactant.

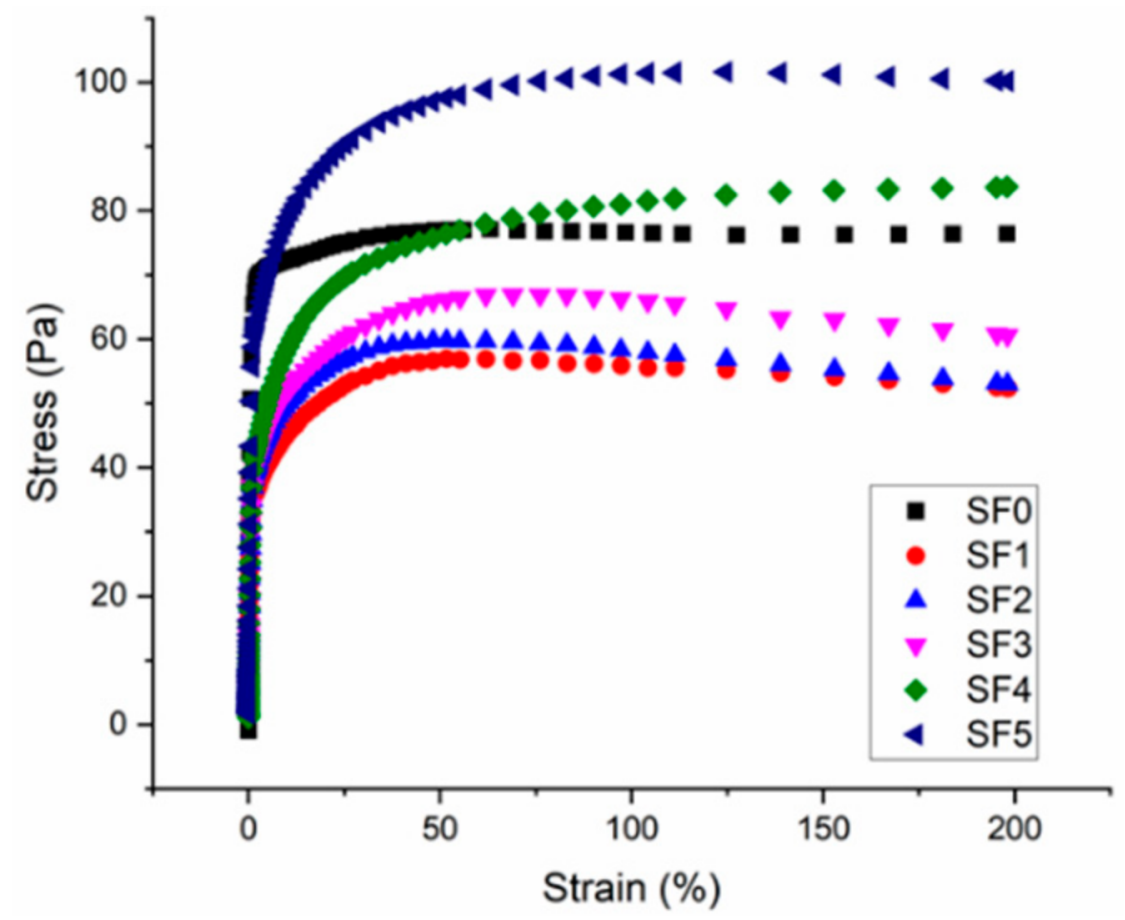

Figure 7. Shear stress versus strain at a constant shear rate of $0.0067 \mathrm{~s}^{-1}$ for CBR gel formulations with $0-5$ wt.\% surfactant.

These results highlight how the gel becomes increasingly elastic when the surfactant concentration is increased. Without surfactant (SF0), the elastic regime is almost nonexistent because the gel is only held together by Van der Waals interactions. Adding surfactant creates new hydrophobic interactions between polyethylene glycol groups and the elastic behavior of the gel extends to higher strain rates.

As shown in Figure 6, even at the minimum of the U-shaped curve of the yield stress versus surfactant concentration, the yield stress remains well above the minimum value (10 Pa) required for optimal use [1,2]. At surfactant concentrations above $4 \mathrm{wt} . \%$, however, the CBR gel becomes more compact, with a yield stress higher than in the absence of surfactant. Thus, although adding more surfactant improves the gel's stability against sedimentation, it also affects the gel's rheological properties.

\subsubsection{Impact of the Surfactant Concentration on the Wetting of a Ceramic Tile}

The presence of a surfactant in a solution increases its wettability on most substrates [22,23]. In decontamination operations, a higher wettability ensures better solubilization and/or sorption of the contamination by/into the gel matrix and thus improves the overall efficiency of the process. Figure 8 shows the contact angle measured on ceramic tiles as a function of the surfactant concentration of the decontaminating solution (i.e., CBR gels without alumina particles), after different storage times. 


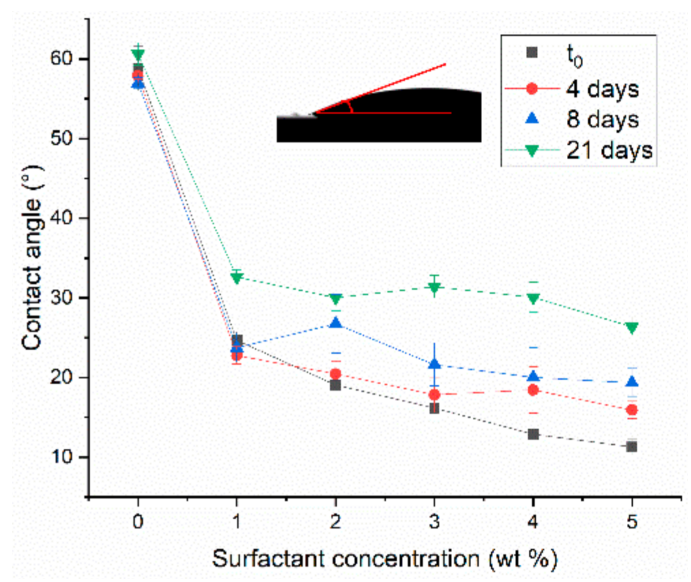

Figure 8. Contact angle between CBR decontaminating solutions with surfactant concentrations of 1-5 wt.\% and a ceramic tile substrate, measured in fresh samples and after 4, 8, and 21 days of storage.

The contact angle decreases substantially between 0 and $1 \mathrm{wt} . \%$ surfactant, and more gradually thereafter. This means that a significant increase in wettability, and thus in the decontaminating efficiency, can be achieved by adding just $1 \mathrm{wt} . \%$ of Pluronic PE 6200 . The same trend is observed for all the fresh and aged samples. The contact angles increase uniformly over time at all surfactant concentrations, suggesting that the surfactant degrades over time in the decontaminating solution and thus the solutions wet the substrate less.

\subsubsection{Estimated Effect of the Surfactant Concentration on the Efficiency of the CBR Gel}

The chemical evolution of the CBR gel was investigated by measuring the concentrations of hypochlorite $\left(\mathrm{ClO}^{-}\right)$and chloride $\left(\mathrm{Cl}^{-}\right)$ions in the decontaminating solution as a function of time (Figure 9). The concentrations are expressed as percentages of the initial values. Their expressions are detailed in Appendix B.
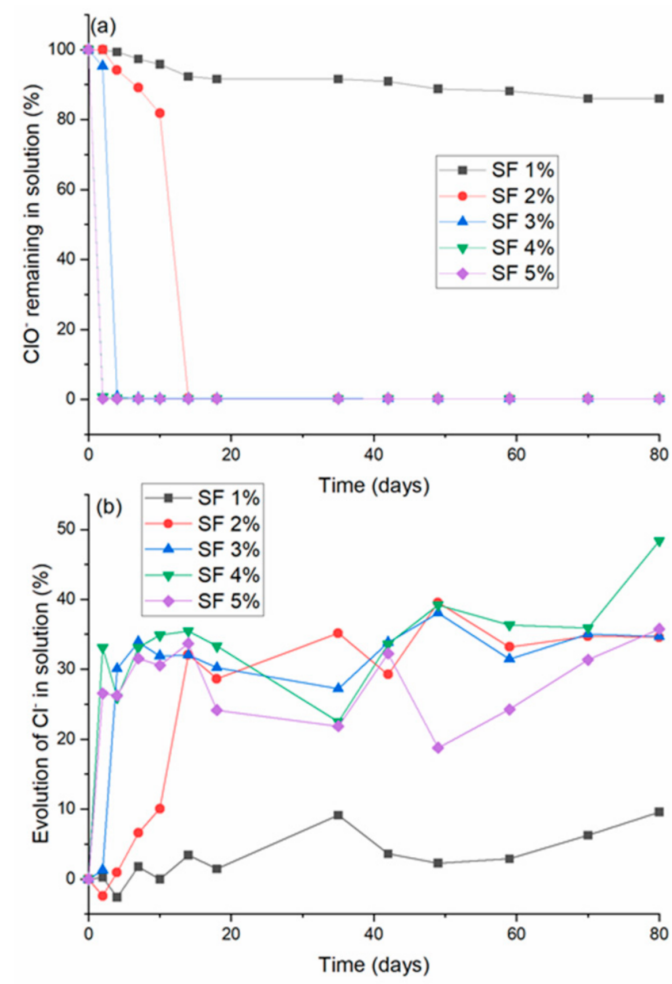

Figure 9. Time evolution of (a) the normalized concentration of hypochlorite ions and (b) the percentage increase in chloride ions in CBR decontaminating solutions with 1-5 wt.\% surfactant. 
Overall, the concentrations of hypochlorite ions decrease over time, while the concentrations of chloride ions generally increase, although the profiles of these trends depend strongly on the surfactant concentration. The decrease in the $\mathrm{ClO}^{-}$concentration is much sharper in the solutions with higher surfactant concentrations, reaching zero in a few days, and the increase in the chloride concentration is likewise faster. These changes reflect the reduction of the hypochlorite ions into chloride ions:

$$
\mathrm{ClO}^{-}(\mathrm{aq}) \rightarrow \mathrm{Cl}^{-}(\mathrm{aq})+\frac{1}{2} \mathrm{O}_{2}(\mathrm{~g})
$$

According to this reaction, the total degradation of the hypochlorite ions should lead to an increase of approximately $35 \%$ in the concentration of chloride ions. This value is reached after 15 days of aging for the SF2 solution and after only 10 days of aging for all the other solutions containing more than $3 \mathrm{wt} \%$ surfactant. In a similar experiment performed in the presence of $\mathrm{NaOH}$ and without surfactant, the concentration of hypochlorite and chloride ions remained practically invariant over time (see Appendix C). This indicates that the surfactant is involved in the reduction of the hypochlorite ions and suggests that the surfactant probably degrades by oxidation of the ether bond by the hypochlorite ions. The surfactant also seems to act as a catalyst for the reduction of the hypochlorite ions.

Consequently, as shown in Figure 8, the higher the surfactant concentration in the solution is, the faster $\mathrm{ClO}^{-}$is reduced into $\mathrm{Cl}^{-}$. This behavior is in agreement with the above interpretations of the rheological data (Figures 2 and 3) and with the evolution of the contact angle of the decontaminating solution on ceramic tiles (Figure 8).

Since the presence of hypochlorite ions is crucial for the gel to be effective against biological contaminants [4], the fact that the concentration of hypochlorite ions decreases substantially over time is not favorable for these applications. An optimal balance must therefore be found, depending on the intended use of the gel, between stabilizing its physicochemical properties (resistance to sedimentation, rheological properties, and substrate wetting) and preserving a sufficiently high decontamination efficiency.

\section{Conclusions}

Vacuumable gels are complex fluids that can be used to efficiently treat contaminated surfaces. Once spread on the surface, the gel absorbs or dissolves the contaminant before drying out, producing only solid waste, such that no effluent post-treatment is required. This technology has been adapted for CBR decontamination, with a highly basic solution containing hypochlorite ions used to inhibit biological and chemical contamination.

In this work, the aging of a CBR gel with 1 wt.\% Pluronic PE 6200 as surfactant was monitored over several months. Results show that the gel only remains stable for a few months, before gradually degrading and altering their rheological properties as a result. Increasing the surfactant concentration improves the resistance of the gel to sedimentation, and the rheological properties remain suitable for spraying. However, because the hypochlorite ions react with the surfactant, their concentration decreases, altering the decontamination efficiency of the gel. To conclude, when the gel is formulated and produced, a balance has to be struck between its long-term efficiency against biological and chemical contaminants and the stability of its physicochemical properties. The optimal choice depends on the intended application and storage possibilities.

Author Contributions: Conceptualization, A.G. and C.L.; Methodology, A.G.; Validation, F.F., C.A., C.P. and N.F.; Formal Analysis, A.G.; Investigation, F.F., C.A. and C.P. and N.F.; Writing Original Draft Preparation, A.G.; Writing Review and Editing, A.G. and C.L.; Visualization, A.G.; Supervision, A.G. and C.L.; Project Administration, C.L.; Funding Acquisition, C.L. All authors have read and agreed to the published version of the manuscript.

Funding: This work was funded by the CEA through the French interministerial R\&D research program on CBRN-E threats.

Institutional Review Board Statement: Not applicable.

Informed Consent Statement: Not applicable. 
Acknowledgments: The authors thank Antoine Leydier for fruitful discussions.

Conflicts of Interest: The authors declare no conflict of interest.

\section{Appendix A}

Description of the Titration Method used to Measure the Concentration of Hypochlorite Ions in the CBR Solutions

Starting with $10 \mathrm{~mL}$ of the solution to be titrated, $20 \mathrm{~mL}$ of a $0.1 \mathrm{M}$ potassium iodide solution and 3-5 drops of $95 \% \mathrm{H}_{2} \mathrm{SO}_{4}$ were added, making the solution turn brown, with potassium iodide in excess. A $0.1 \mathrm{M}$ solution of sodium thiosulfate was then added dropwise, making the mixture progressively become yellow. Just before equivalence, a few drops of a starch solution were added to make the solution turn black, to better identify that equivalence and the titration was stopped when the solution suddenly became light pink. The concentration of hypochlorite ions was deduced from the following redox equations:

$$
\begin{gathered}
\mathrm{ClO}^{-}{ }_{(\mathrm{aq})}+2 \mathrm{H}^{+}{ }_{(\mathrm{aq})}+2 \mathrm{e}^{-}=\mathrm{Cl}^{-}{ }_{(\mathrm{aq})}+\mathrm{H}_{2} \mathrm{O}_{(\mathrm{l})} \\
2 \mathrm{I}^{-}{ }_{(\mathrm{aq})}=\mathrm{I}_{2(\mathrm{aq})}+2 \mathrm{e}^{-} \\
\mathrm{ClO}^{-}{ }_{(\mathrm{aq})}+2 \mathrm{H}^{+}{ }_{(\mathrm{aq})}+2 \mathrm{I}^{-}{ }_{(\mathrm{aq})}=\mathrm{I}_{2(\mathrm{aq})}+\mathrm{Cl}^{-}{ }_{(\mathrm{aq})}+\mathrm{H}_{2} \mathrm{O}_{(\mathrm{l})} \\
\mathrm{I}_{2(\mathrm{aq})}+2 \mathrm{e}^{-}=2 \mathrm{I}^{-}{ }_{(\mathrm{aq})} \\
2 \mathrm{~S}_{2} \mathrm{O}_{3}{ }^{2-}{ }_{(\mathrm{aq})}=\mathrm{S}_{4} \mathrm{O}_{6}{ }^{2-}{ }_{(\mathrm{aq})}+2 \mathrm{e}^{-} \\
\mathrm{I}_{2(\mathrm{aq})}+2 \mathrm{~S}_{2} \mathrm{O}_{3}{ }^{2-}{ }_{(\mathrm{aq})}=2 \mathrm{I}^{-}{ }_{(\mathrm{aq})}+\mathrm{S}_{4} \mathrm{O}_{6}{ }^{2-}{ }_{(\mathrm{aq})}
\end{gathered}
$$

The hypochlorite ion concentration was finally calculated as follows:

$$
\begin{gathered}
\mathrm{n}\left(\mathrm{ClO}^{-}\right)_{\text {consumed }}=\mathrm{n}\left(\mathrm{I}_{2}\right)_{\text {producted }}=\mathrm{n}\left(\mathrm{S}_{2} \mathrm{O}_{3}{ }^{2-}\right) / 2 \\
{\left[\mathrm{ClO}^{-}\right] \times \mathrm{V}(\text { solution })=\left(\left[\mathrm{S}_{2} \mathrm{O}_{3}{ }^{2-}\right] \times \text { Veq }\right) / 2} \\
{\left[\mathrm{ClO}^{-}\right]=10 \times\left(\left[\mathrm{S}_{2} \mathrm{O}_{3}{ }^{2-}\right] \times \text { Veq }\right) / 2 \mathrm{~V}(\text { solution })}
\end{gathered}
$$

\section{Appendix B}

Expression used for the calculation of the $\mathrm{ClO}^{-}$remaining in solution and of the percentage of chloride concentration increase in solution with time

$$
\mathrm{ClO}^{-} \text {remaining in solution }(\%)=100 \times \frac{\left[\mathrm{ClO}^{-}\right]_{\text {in solution }}}{\left[\mathrm{ClO}^{-}\right]_{\text {initial }}}
$$

Percentage of $\left[\mathrm{Cl}^{-}\right]$increase in solution $(\%)=100 \times \frac{\left[\mathrm{Cl}^{-}\right]_{\text {in solution }}-\left[\mathrm{Cl}^{-}\right]_{\text {initial }}}{\left[\mathrm{Cl}^{-}\right]_{\text {initial }}}$

\section{Appendix C}

Time evolution of the percentage concentrations of hypochlorite and chloride ions in a $1 \mathrm{M} \mathrm{NaOH}$ solution.

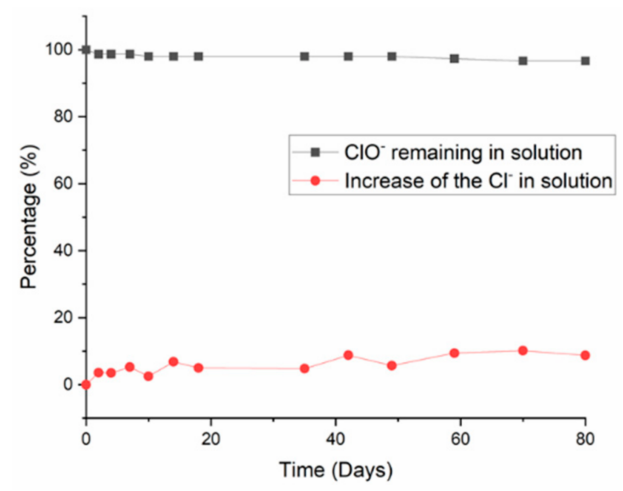

Figure A1. Concentrations of hypochlorite and chloride ions as a function of time in a $1 \mathrm{M} \mathrm{NaOH}$ solution, expressed as percentages of the initial values. 


\section{References}

1. Faure, S.; Fuentes, P.; Lallot, Y. Vacuumable Gel for Decontaminating Surfaces and Use Thereof. World Patent WO 2007039598, 12 April 2007.

2. Ludwig, A.; Goettmann, F.; Frances, F.; Legoff, C.; Tanchou, V. Oxidizing Alkaline Biodecontamination Gel and Surface Biodecontamination Method Using Said Gel. World Patent WO 2014154818, 10 February 2014.

3. Gossard, A.; Lepeytre, C. An innovative green process for the depollution of $\mathrm{Cr}(\mathrm{VI})$-contaminated surfaces using $\mathrm{TiO}_{2}$-based photocatalytic gels. J. Environ. Chem. Eng. 2017, 5, 5573-5580. [CrossRef]

4. Lepeytre, C.; Frances, F.; Charvolin, M.S.; Ludwig, A.; Le Toquin, E.; Comoy, E.; Grandjean, A.; Gossard, A. Colloidal gel as an efficient process to treat Chemical, Biological, Radiological (CBR) and prion contaminated solid surfaces. Chem. Eng. Sci. 2021, 246, 116957. [CrossRef]

5. Castellani, R.; Poulesquen, A.; Goettmann, F.; Marchal, P.; Choplin, L. A topping gel for the treatment of nuclear contaminated small items. Nucl. Eng. Des. 2014, 278, 481-490. [CrossRef]

6. Castellani, R.; Poulesquen, A.; Goettmann, F.; Marchal, P.; Choplin, L. Efficiency enhancement of decontamination gels by a superabsorbent polymer. Colloid Surf. A-Physicochem. Eng. Asp. 2014, 454, 89-95. [CrossRef]

7. Yoon, S.B.; Kim, C.K.; Jung, C.H.; Choi, B.S.; Choi, W.K.; Lee, K.W.; Moon, J.K. Effect of Alkyl Alcohol on Viscosity of Silica-Based Chemical Gels for Decontamination of Radioactive Contaminations. Asian J. Chem. 2013, 25, 7023-7027. [CrossRef]

8. Jung, C.H.; Moon, J.K.; Choi, W.K. Effect of Chemical Formulations for Uranium Decontamination by Chemical Gels. Asian J. Chem. 2016, 6, 1285-1287. [CrossRef]

9. Lemesre, L.; Frances, F.; Grandjean, A.; Gossard, A. Hybrid colloidal suspensions tailored as gels to remove radioactive bitumen stains. J. Environ. Manag. 2019, 232, 660-665. [CrossRef] [PubMed]

10. Derjaguin, B.; Landau, L.D. Theory of the stability of strongly charged lyophobic sols and of the adhesion of strongly charged particles in solutions of electrolytes. Acta Physicochimica U.R.S.S. 1941, 14, 633-662. [CrossRef]

11. Liang, Y.; Hilal, N.; Langston, P.; Starov, V. Interaction forces between colloidal particles in liquid: Theory and experiment. Adv. Colloid Interface Sci. 2007, 134-35, 151-166. [CrossRef] [PubMed]

12. Verwey, E.J.W.; Overbeek, J.T.G.; Nes, K.V. Theory of the Stability of Lyophobic Colloids: The Interaction of Sol Particles Having an Electric Double Layer; Elsevier Publishing Company: New York, NY, USA, 1948.

13. Herschel, W.M.; Bulkley, R. Measurement of consistency as applied to rubber-benzene solutions. Proc. Am. Soc. Test. Mater. 1926, $26,621-633$.

14. Ovarlez, G.; Cohen-Addad, S.; Krishan, K.; Goyon, J.; Coussot, P. On the existence of a simple yield stress fluid behavior. J. Non-Newton. Fluid Mech. 2013, 193, 68-79. [CrossRef]

15. Xu, J.H.; Li, J.Y.; Cao, J.G. Effects of fumed silica weight fraction on rheological properties of magnetorheological polishing fluids. Colloid Polym. Sci. 2018, 296, 1145-1156. [CrossRef]

16. Gossard, A.; Frances, F.; Aloin, C. Rheological properties of $\mathrm{TiO}_{2}$ suspensions varied by shifting the electrostatic inter-particle interactions with an organic co-solvent. Colloid Surf. A-Physicochem. Eng. Asp. 2017, 522, 425-432. [CrossRef]

17. Zhou, Z.W.; Scales, P.J.; Boger, D.V. Chemical and physical control of the theology of concentrated metal oxide suspensions. Chem. Eng. Sci. 2001, 56, 2901-2920. [CrossRef]

18. Castellani, R.; Poulesquen, A.; Goettmann, F.; Marchal, P.; Choplin, L. Ions effects on sol-gel transition and rheological behavior in alumina slurries. Colloid Surf. A-Physicochem. Eng. Asp 2013, 430, 39-45. [CrossRef]

19. Sakar-Deliormanli, A.; Polat, H.; Ciftcioglu, M. Alumina/water suspensions in the presence of PEO-PPO-PEO triblock copolymers. J. Eur. Ceram. Soc. 2004, 24, 3063-3072. [CrossRef]

20. Bleta, R.; Alphonse, P.; Pin, L.; Gressier, M.; Menu, M.J. An efficient route to aqueous phase synthesis of nanocrystalline gammaAl2O3 with high porosity: From stable boehmite colloids to large pore mesoporous alumina. J. Colloid Interface Sci. 2012, 367, 120-128. [CrossRef] [PubMed]

21. Fulvio, P.F.; Brosey, R.I.; Jaroniec, M. Synthesis of Mesoporous Alumina from Boehmite in the Presence of Triblock Copolyme. ACS Appl. Mater. Interfaces 2010, 2, 588-593. [CrossRef] [PubMed]

22. Zhou, L.; Yang, S.Y.; Hu, B.; Yuan, Z.L.; Wu, H.; Yang, L.J. Evaluating of the performance of a composite wetting dust suppressant on lignite dust. Powder Technol. 2018, 339, 882-893. [CrossRef]

23. Wang, H.T.; Wei, X.B.; Du, Y.H.; Wang, D.M. Effect of water-soluble polymers on the performance of dust-suppression foams: Wettability, surface viscosity and stability. Colloid Surf. A-Physicochem. Eng. Asp. 2019, 568, 92-98. [CrossRef] 